\section{Tracking Particles by Lasers}

\author{
R.A. Stern, Lausanne \\ (EPFL Centre de Recherches en Physique des Plasmas)
}

\begin{abstract}
Particle transport can be studied by determining changes in the ground state population following the raising of a selected source group into a long-lived metastable state.
\end{abstract}

Optical tagging comprises a class of new measurement techniques which permit several basic processes in gases and plasmas to be diagnosed directly for the first time. These remote, non-perturbing methods are useful in characterizing transport (diffusion, convection) and field-particle interactions (heating, trapping). In addition to high space- and time-resolution, they can trace the details of the particle velocity (Boltzmann) distribution with great detail. A novel aspect is that optical tagging has a builtin "memory" and therefore provides information on causality. Thus the trajectory of particles through phase space, including their direction, is mapped out. By these means a complete kinetic description of gaseous processes, applicable in atomic, fluid and plasma physics, can be approached.

Two quantum processes: velocity-selective optical pumping, and laser-induced fluorescence, underlie optical tagging. First, optical pumping is used to tag (label) a group of particles at a "source" point in phase space. That is, by means of radiation, one selectively assigns to the particles a quantum property which distinguishes them from the surrounding medium. Second, the migration of the tagged particles from the source to a "field point" is traced using laser-induced fluorescence. Both processes derive from the fact that atoms, molecules and ions can exist in discrete quantum states with finite lifetimes, which are interconnected with states of the radiation field (photons).

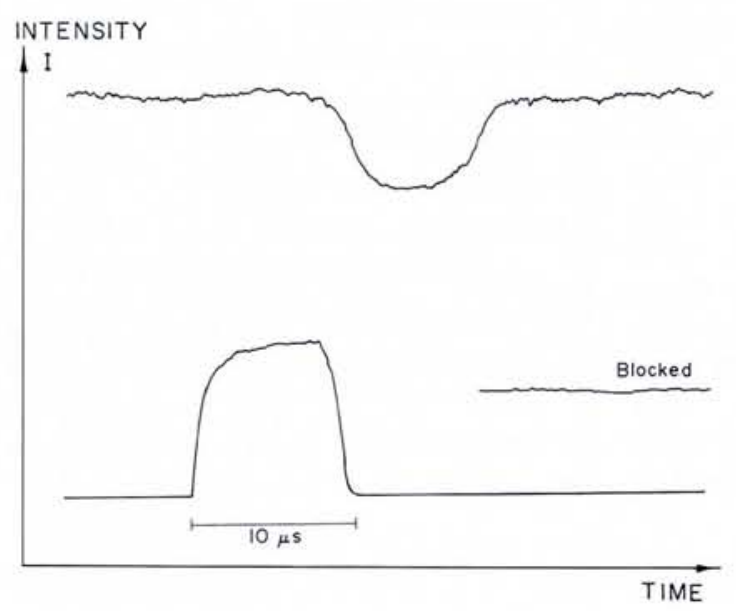

These processes and the principal physical factors involved are described below, in connection with their use in measuring ion transport in a plasma. Consider a plasma containing singlyionized barium ( $\mathrm{Ba}$ II). This is practical because $\mathrm{Ba}$ II is a common, easily-handled ion whose quantum scheme allows strong (dipole) transitions from the ground state to be excited by radiation in the visible part of the spectrum, where tunable lasers are readily available. In our example, a "pump" beam of blue-green light at $4934 \AA$ induces transitions from the $6^{2} \mathrm{~S}_{1 / 2}$ ground state of $\mathrm{Ba}$ II to the $6^{2} \mathrm{P}_{1 / 2}$ excited state with high efficiency, since the photon energy ( $\sim 3 \mathrm{eV})$ precisely equals the energy difference between the states (resonance fluorescence).

The excited state can decay spontaneously to only two levels: the ground state, and the metastable state $5^{2} D_{3 / 2}$. The Einstein $A$ coefficients, describing the spontaneous transition probabilities for the two decay channels, have the values $A_{1}=95 \times 10^{6} \mathrm{~s}^{-1}$ and $A_{2}=33$ $\times 10^{6} \mathrm{~s}^{-1}$ respectively, corresponding to a lifetime $\left(A_{1}+A_{2}\right)^{-1}=7.7 \times 10^{-9}$ for the excited state, and a branching ratio $A_{1} /\left(A_{1}+A_{2}\right)=0.74$. That is, within $10^{-8} \mathrm{~s}$ following their excitation, via the spontaneous decay channel, nearly three out of every four ions excited from the ground-state return to it, but the fourth is pumped into the metastable state. This process tends to alter the distribution of ions over quantum states in a specific, irreversible manner: the

Fig. 1 - Experimental results in Optical Tagging: measurement of ion diffusion using pulse tagging; I - light intensity against Time. Lower trace: Excitation by pump laser beam pulse applied at source. Upper trace: Delayed response, laser-induced fluorescence detected at field point. Transition $6^{6} S_{1 / 2}-6^{2} P_{1 / 2}$ in Ba II, $4934 \AA$. ground-state population becomes depleted, while the metastable population is enhanced.

The metastable state lifetime, about $1 / 4 \mathrm{~s}$, is very long in comparison with the mean life of plasma ions: for instance, the ratio of a plasma device's dimensions, say $100 \mathrm{~cm}$, to the ion speed, typically $10^{5} \mathrm{~cm} / \mathrm{s}$ or higher is less than $10^{-3} \mathrm{~s}$. Thus using optical pumping the experimenter can prepare, at the time and position of his choice, a distinct quantum configuration which is carried along by the ions and can persist during their migration across the plasma. In addition, by using narrow-band pumping radiation, the initial velocity of these ions can be pre-determined.

To identify the presence of these "tagged" ions at other positions and times - the diagnosed or field point use is made of laser-induced fluorescence. This is a technique of increasing use in atomic, nuclear, surface, fluid and plasma physics. In our example, the axis of a "search" beam at $4934 \AA$ (the pump wavelength), and the optical axis of a lens, are aligned so that they intersect at the field point. A wavelength filter and a detector behind the lens select and monitor the radiation at $4934 \AA$ emitted during decay from the excited to the ground states. This intensity is proportional to the density of ground-state ions at the field point. A fraction of these ions represents the flux of particles from the source.

The change in ground-state population caused by optical pumping at the source point will induce a corresponding change in the flux and consequently in the radiation intensity emitted at the field point. This radiative response contains the principal information describing ion transport between the two points.

An illustration of actual measurements is shown in Fig. 1. The output of a $\mathrm{CW}$ tunable laser, using a variant of the dye coumarin pumped by the UV line of a krypton laser, is tuned to $4934 \AA$ and split into two beams. These are positioned to traverse a low-density $\left(10^{9} \mathrm{~cm}^{-3}\right)$ Ba II plasma contained in a magnetic field along parallel axes normal to the field and spaced $2 \mathrm{~cm}$ apart. One beam, the pump, is chopped to yield pulses with short rise- and fall-times $(0.1 \mu \mathrm{s})$. The second, diagnostic or search beam is on continuously. The ordinate in Fig. 1 plots the laser-induced fluorescence intensity $I$ at a field point on the search beam axis. As shown in the figure, 1 varies with time in a manner distinct from the pump beam pulse. During the first $9 \mu$ s after the pump beam is on, I 
does not vary. Then it begins to decrease at a rate much slower than the rise-time of the pump beam, reaches a stationary level at $15 \mu$ s and eventually increases, slowly again, to its original level. This delayed response is interpreted as follows: the ions tagged at the source point and which migrate to the field point turn out to have a velocity distribution characterized by a mean speed as well as a nearly Gaussian spread. The fastest ions arrive first, causing the initial decrease in I; their speed is roughly $2 \mathrm{~cm} / 9 \mu \mathrm{s}$, or 2.2 $\times 10^{5} \mathrm{~cm} / \mathrm{s}$. The mean speed corresponds to the stationary level, $2 \mathrm{~cm} / 15 \mu \mathrm{s}$ or $1.3 \times 10^{5} \mathrm{~cm} / \mathrm{s}$. Evidently the timevariation of $l$ in between these extrema, traces out in detail the entire velocity distribution for the ions which migrate from source to field point at the time of the measurement.

This interpretation is checked out using independent and much more laborious methods. It is remarkable that optical tagging can yield such details concerning the ion transport without recourse to dispersive optics (interferometers), using only broad-band laser radiation, and with the beams positioned at right angles to the ion motion, so that the pump beam does not select ion speeds at the source point. In unfolding such data, several factors must be considered. First, in addition to spontaneous decays, induced transitions - proportional to the product of the Einstein $B$ coefficients, the $g$-factors of the states, and the radiation intensity - also affect the rate at which the quantum state population changes within the pump beam. Collisional processes and transittime effects can also come into play. In general, a system of rate equations is needed to describe the evolution of the population, and the velocity distribution of the tagged ions may end up different from that of the original, un-pumped ions. Second, during transit from source to field point, collisional or radiative transitions could alter the tagged quantum population causing a loss of "memory". Analytical expressions for handling these cases are on hand, principally from the literature on laser-induced fluorescence.

It is simplest, however, when the spontaneous decay rate is much larger than all other terms, and the distance from source to field point is smaller than the quantum mean free path. This is the case in the example given here, where the pump intensity and beam diameter are kept at a minimum, and the ion velocities, electron density and temperature are low. As a result the variation in / with time represents directly the velocity dis- tribution of the unperturbed diffusing ions.

Three other parameters are important. First, the radiation must interact directly with the particles, rather than with the plasma collective modes. The latter become significant when the plasma dielectric function approaches zero, e.g. if the light frequency approaches the plasma frequency $\left(4 \pi n e^{2} / m\right)^{1 / 2}$, where $n$ is the charge density, and $e$ and $m$ are the electron charge and mass (in an electron-ion plasma). For radiation in the visible, $n$ has to be less than $10^{21} \mathrm{~cm}^{-3}$, a value fortunately much beyond most laboratory plasmas.

Second, the pump intensity should be sufficiently intense to create an appreciable change in the quantum state densities Semi-classically, the Einstein $A$ and $B$ coefficients representing stimulated transitions satisfy the detailed-ba- lance relation $B=A C \lambda^{2} / 8 \pi$ per unit energy, where $c$ is the speed of light and $\lambda$ is the radiation wavelength. This relationship is equivalent to a cross-section for resonant excitation with a peak value $\sigma \cong \lambda^{2} / 8 \pi$. In the visible, $\sigma$ is roughly $10^{-10} \mathrm{~cm}^{2}$, a very large value (the electron Thomson scattering cross-section is about $10^{15}$ smaller). A $10 \mathrm{~mW}$ beam in the visible ( $3 \mathrm{eV}$ photons) with diameter $1 \mathrm{~mm}$, corresponds to a photon flux of 2 $\times 10^{18} \mathrm{~cm}^{-2} \mathrm{~s}^{-1}$. The product of crosssection, radiation flux and $g$-factors is therefore of the order of $10^{8} \mathrm{~s}^{-1}$, indicating that the laser-induced excitation rate can be close to the excited-state lifetime for the case above. In general, a low-power laser - narrow enough in wavelength just to cover the particle absorption Doppler width (roughly $0.05 \AA$ in our example) — will suffice to saturate the transition. Such spectral intensi-

Re-announcement

\section{The Technical University of Denmark Professorship in Optics}

Subject to confirmation of the granting authorities, a professorship in Optics is to be filled in 1984 in order to strengthen the research within one or several of the following subject groups:

\section{a) Coherent Optics}

Research concerning coherence-properties of light (interference, photon statistics) including applications, for example, in connection with holography, holographic interferometry, anemometry, optical data processing, etc.

\section{b) Interaction between light and matter}

Research concerning absorption, spontaneous and stimulated emission, the transmission and scattering of light and various non-linear optical effects.

\section{c) Optoelectronics}

Application-oriented research concerning the physics of optoelectronic components such as lasers, electro- and acoustic optical components, integrated optics, optical fibres, etc.

\section{d) Optical Systems}

Design and evaluation of optical systems.

The applicant should have shown a high degree of scientific maturity and have proven research capability in one or preferably several of the above subject groups. He must be able to undertake teaching of elementary and advanced physics and to serve as supervisor for students majoring in physics as well as for Ph.D. studies. Thus the pedagogical qualifications are also important for the judgment of the applicants.

Further information about the professorship, the salary, the conditions of tenure, and the procedure of the judging committee etc. may be obtained from senior lecturer Flemming Damhus Pedersen,

The Technical University of Denmark, Building 303, DK-2800 Lyngby (Telephone: (45) (2) 883699 , ext. 4337).

The application should include curriculum vitae, relevant publications, if possible in four copies, and a detailed account of teaching experience. It is to be addressed to Her Majesty the Queen of Denmark and sent to the

Rector of the Technical University of Denmark,

Building 101 A, DK-2800 Lyngby, Denmark.

Applications must be received not later than 31 July, 1984. 
Fig. 2 - Velocity Selection in Optical Tagging: experimentally measured Boltzmann distribution functions of tagged ions in a magnetic field; 1 - light intensity against Velocity. Parameter $\Phi$ : axial separation along magnetic field lines between pump and search laser beams, normalized to $v / \omega_{c}$. With $v=$ mean diffusion velocity of ions along field and $\omega_{c}$ $=$ ion cyclotron frequency. Trace a: $\Phi=\pi$; dotted lines outline hyperfine structure obtained by long-term integration in this velocity region. Trace $b: \Phi=0$ and $\pi$. Trace c: plot of Gaussian equiva-

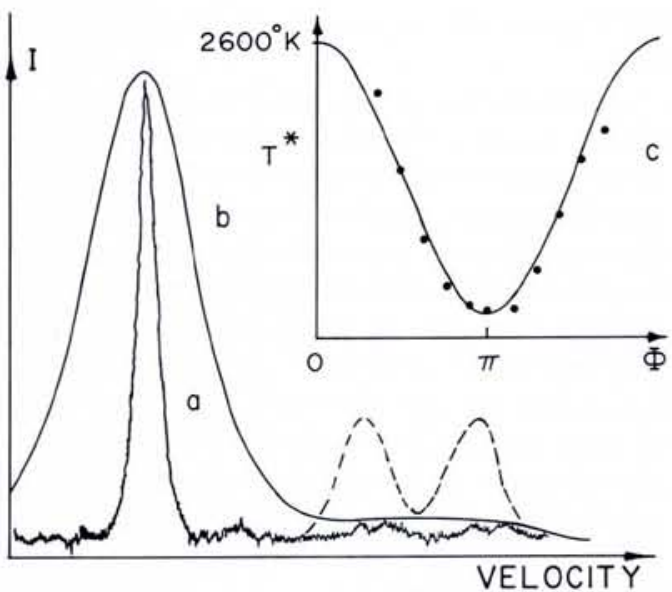

lent temperature $T^{*}$ of Boltzmann distribution functions as a function of axial separation $\Phi$. Measurements on a single Zeeman component of transition $6{ }^{2} S_{1 / 2}-6^{2} P_{1 / 2}$ in $B$ a II, $4934 \AA$.

ties are easily available in tunable lasers. Using more intense radiation does not alter the quantum population when used in the pump beam, and will power-broaden the observed distribution if used in the search beam.

Third, signal/noise ratio at the field point must be adequate. An upper limit on fluorescence is obtained in an idealized two-level quantum system, which saturates with the populations of excited and depleted ground state at roughly equal values. The signal available then is the product of half the tagged particle density, the diagnosed volume, and the spontaneous decay rate. For our example, the last two factors are approximately $(1 \mathrm{~mm})^{3} \times 10^{8} \mathrm{~s}^{-1}$, or $10^{5}$ photon $/ \mathrm{s}$ for each ion $/ \mathrm{cm}^{3}$ diffusing to the field point. This flux is emitted into $4 \pi$ steradians, of which only a fraction, $10 \%$ at most, can be remotely intercepted via finite f-number optics. One notes however that light rates of the order of 1 photon/s or less can be detected using signal processing. Noise terms competing with the signal include emission at the observed wavelength, from spontaneous transitions occurring in the medium within the field of view of the detection optics. In the example given, the electron density and energy are so low that the medium is dark, and this noise term remains negligible.

Many alternative experimental configurations are possible. For instance, using the appropriate wavelength filter at the field-point one can monitor the laser-induced fluorescence via decay from the excited to the metastable state at $6497 \AA$. This has a slower rate and provides less signal, but is so far removed from the laser wavelength that accidentally scattered "elastic" laser light can be blocked out. Another scheme uses a separate search laser. This can excite any allowed transition, including ones with higher decay rates. The two- iaser system makes possible a new signal mode. If tuned to a transition which has the metastable state as the lower level, it "interrogates" the metastable population and therefore produces an increase in the signal intensity (bright signal) when the density enhanced by the pump beam reaches the field point. This can enhance signal/noise appreciably. The dark - and bright signal schemes are also known by the names of the quantum optics pioneers Kastler and Dehmelt.

Major sophistication in optical tagging is afforded by the use of narrow-band radiation in the pump or search beams. The Doppler principle requires that radiation at $\lambda$ interacts resonantly only with particles whose velocity component $v$ along the laser beam axis satisfies the formula $\lambda \cong \lambda_{0}(1+v / c)$, where $\lambda_{0}$ is the resonant wavelength for particles at rest. For instance, single-mode scanning lasers whose frequency bandwidth is less than $10^{6} \mathrm{~Hz}$ can tag and diagnose sub-groups of particles with velocity ranges $\Delta v \leq 10^{6} \lambda \approx 10^{2}$ $\mathrm{cm} / \mathrm{s}$, using visible radiation. By these means, velocity/velocity correlation measurements in phase-space can be made in great detail. A simple version of such an experiment uses a single narrow-band laser split into two beams, on the same Ba II plasma as above. Now the separation betweeen the beams is varied along the magnetic field lines, and at each position the laser wavelength is scanned over the Doppler ion velocity profile. The pump beam is modulated, and the corresponding modulated component $I$ of the laser-induced fluorescence is plotted as in Fig. 2. It represents the velocity distribution of ions which diffused to the field point, were tagged at the source, but retained their velocity component along a specific axis normal to the magnetic field. A very large systematic variation in the velocity distribu- tion function is found as a function of the distance. The reason is that, in a magnetic field, the velocity and position of a charged particle are coupled by the Lorentz force, resulting in spiral Larmor trajectories with a unique periodicity and $a$ radius that is a function of the transverse velocity.

The two-laser scheme selects on both the velocity and position of the tagged ions, and only a narrow sub-class can satisfy the double requirement. A simple analysis verifies this relationship, predicting the effective "temperature" $T^{*}$ as a function of the normalized distance $\Phi$ along the magnetic field, as shown in trace $c$. This varies from a maximum of $2600 \mathrm{~K}$ to a minimum of $60 \mathrm{~K}$ over a distance corresponding to one-half a complete Larmor orbit. This velocity selection for in-situ particles can be useful in eliminating Doppler widths which obscure spectroscopic features such as the Zeeman effect and hyperfine structure. The details (dashed line) in the trace show the enhanced hyperfine detail, which otherwise require accelerator techniques for resolution.

Optical tagging has also been used to measure the trajectories in phase space of ions trapped and de-trapped by cyclotron waves, to plot the cross-magnetic field ion diffusion in unstable and heated plasmas, and also in polarization spectroscopy and atomic beam magnetic resonance studies. As an example in the last-named fields, sodium atoms with initially random spins are optically spin oriented by a circularily-polarized pump laser beam at the principal resonance $3 S_{1 / 2}(F=2)-3 P_{1 / 2}(F=1)$. They diffuse across a magnetic field and are detected by a search beam, split off the same laser, displaced $1 \mathrm{~cm}$ across the field. The atoms precess during their transit, leading to changes in the strength of their interaction with the linearily-polarized search beam. The precession, as well as the effect of collisions with a buffer gas of variable density, can therefore be measured as a function of atomic velocity. Evidently, entire classes of measurement which classically required uniform magnetic fields can now be carried out, with much enhanced detail, using lasers instead.

\section{BIBLIOGRAPHY}

For a detailed application to atoms, see:

Bichi P., Moi L., Savino P. and Zambon B., "Measurement of the Diffusion Coefficient of Oriented $\mathrm{Na}$ Atoms in Different Buffer Gases" II Nuovo Cimento 55 B (1980) 1.

The application to plasma ions appears in:

Stern R.A., Hill D.N. and Rynn N., "Direct Ion Transport Measurement by Optical Tagging" Physics Letters 93 A (1983) 127. 